\title{
¿NIÑA O NIÑO? \\ LA INCERTIDUMBRE DEL SEXO Y EL GÉNERO EN LA INFANCIA
}

\author{
Eva Alcántara \\ Universidad Autónoma Metropolitana
}

\section{RESUMEN}

Es notable la creciente inquietud en torno a la incertidumbre del sexo y género en los primeros años de vida. Mediante el análisis de tres casos atravesados por el tema de la infancia y por los discursos modernos del sexo y el género, en este artículo se discute sobre las transformaciones que estos factores introducen en las condiciones de producción de la identidad sexuada. Las indagaciones científicas sobre el sexo, las teorías psicológicas del desarrollo del género, la política de gradual apertura a espacios posibles de ciudadanía, los movimientos de derechos humanos y la difusión de nuevas narrativas en medios masivos de comunicación, son algunos elementos que reconfiguran en la actualidad las preguntas y las respuestas acerca de quién y qué es un niño o una niña.

PALABRAS CLAVE

Identidad de género, sexo, infancia, intersexualidad, transexualidad

\section{ABSTRACT}

It is notable the increasing interest and quandary surrounding issues of sex and gender during the first years of life. In this paper we analyzed three cases in which the childhood is intertwined with modern discourses of sex and gender. The changes of the conditions in which the sexual identity is produced are exposed. Scientific research regarding sex, psychological theories of gender development, the politics that are gradually opening spaces to different possibilities of citizenship, human rights movements and the dissemination of new narratives through the media, are just a few elements that nowadays 
reconfigure the questions and answers about who and what is a boy or a girl.

KEY WORDS

Gender identity, sex, childhood, intersex, transexuality 


\section{INTRODUCCIÓN}

Cada vez es más frecuente que durante los primeros años de vida, el cuerpo, la sensibilidad, los comportamientos y las formas de jugar sean objeto de vigilancia y evaluación. Gradualmente la infancia se ha configurado como un tema de interés. La vida cotidiana de niños y niñas experimenta cambios en consonancia con otras transformaciones históricas, los cuales no siempre son fácilmente perceptibles. No obstante que en la actualidad niños y niñas aparecen como uno de los focos primordiales de las políticas públicas, los discursos y las instituciones, rara vez se conocen y consideran sus particularidades. Las transformaciones en curso no son uniformes ni estables. Hoy en día es posible distinguir opiniones contradictorias y diferentes estrategias de intervención sobre el sexo y el género durante la infancia.

Michel Foucault (2000 [1976]) señaló que durante el siglo XVIII, uno de los grandes conjuntos estratégicos a partir de los cuales se consolidó el dispositivo de sexualidad fue la multiplicación de intervenciones y controles enfocados en los primeros años de la vida. Fue entonces cuando se amplificó la atención a las menores manifestaciones del sexo en el niño. Philippe Ariès estudió con amplitud cómo progresivamente apareció en el curso de la historia la figura del niño, al tiempo que surgió durante el siglo XIX el concepto de "infancia" y con ello el desarrollo de teorías específicas que encerraron en lógicas particulares - etapas o periodos - la vida del ser humano. Ariès caracterizó el siglo XX por un "sentimiento bifronte [hacia el infante]: de un lado solicitud y ternura, una especie de forma moderna de mimar, y del otro, también solicitud, pero con severidad: la educación" (Ariès, 1986: 14). En las últimas décadas es cada vez más evidente que el núcleo familiar y la institución escolar — espacios que determinaban primordialmente la subjetividad al comienzo de la vida — son permeables a discursos y lógicas que quiebran sus propios ritmos. Hoy en día estas instituciones forman parte de una red extensa que en torno a la infancia detona sensibilidades, genera mercados de consumo, moviliza discursos de derechos y transforma políticas institucionales (Bustelo, 2011).

A partir de estas reflexiones, he seleccionado tres casos actuales por los que se atraviesan el tema de la infancia con los discursos modernos del sexo y del género. Me interesa explorar la trayectoria y las articulaciones que han dado lugar a los discursos estatales y las prácticas de intervención institucionales en torno al sexo y el género en la infancia. Estos casos requerirían de un análisis particular mucho más específico, pero en este texto el conjunto nos abrirá una panorámica de las transformaciones en curso que buscan incidir en la configuración de la identidad - el sexo y el género- especialmente durante los primeros años de la vida.

SOBRE EL GESCHLECHTY Y EL SEXO REGISTRAL EN CASOS DE AMBIGÜEDAD GENITAL

En mayo de 2013 el gobierno alemán aprobó una ley para omitir en algunos casos el llenado de la casilla

1 Geschlecht es la palabra alemana para registrar la asignación sexual del recién nacido. El término no separa el sexo del género, ya que en el idioma alemán no hay una división entre características biológicas y simbolización cultural [agradezco a Brigitte Rigther su asesoría del idioma alemán]. 
Geschlecht, mismo que registra la asignación sexual al nacimiento. La reforma de ley alemana contempla la posibilidad de que un recién nacido, catalogado con ambigüedad de genitales, sea registrado como masculino, como femenino o, bien, dejando vacía la casilla. Dejar vacía la casilla implica que el sexo civil queda no determinado en el certificado de nacimiento. Para dejar en blanco o registrar un cambio en la casilla Geschlecht es indispensable un certificado médico que lo avale (DB, 2013).

La noticia fue ampliamente difundida en medios de comunicación a través de programas de radio y televisión, diarios, sitios web y redes sociales, y logró tener una gran presencia global. La traducción al español fue infortunada y se difundió la falsa versión de que Alemania abría una opción para registrar un tercer sexo al nacimiento, lo cual levantó gran polémica; aún hoy es posible encontrar comentarios en Facebook y notas informativas en páginas electrónicas de periódicos donde el malentendido persiste.

Explica Mauro Cabral, activista trans e intersex argentino, que la reforma a la ley alemana debe comprenderse en consonancia con un grupo de medidas jurídicas recientes que buscan el reconocimiento legal de formas de vida que exceden la lógica binaria de la diferencia sexual. Entre estas reformas Cabral señala el fallo de la Corte Suprema de Nepal, el fallo de la Corte Suprema de la India, la Ley de Identidad de Género aprobada por el Senado argentino y el pronunciamiento de la Corte Suprema de Australia, que dio como resultado el reconocimiento del derecho de Norrie a ser legalmente registrada como una persona de sexo no específico. A decir de Cabral, medidas como éstas contribuyen a "ampliar el horizonte de la lucha por el reconocimiento legal de la identidad de género" (Cabral, 2014: 200). No obstante, aunque la reforma alemana se anunció como una medida progresista que intenta considerar la existencia de cuerpos distintos, integrantes de la comunidad intersex se mostraron en desacuerdo con la modificación. La comunidad intersex advirtió que omitir el sexo de registro en el documento de nacimiento crea un estatus discriminatorio ya que estigmatiza a los bebés que pretende proteger al marcarlos con la indeterminación del sexo en sus documentos de registro; además, la ley alemana continúa dejando en manos del médico el poder de decidir sobre la determinación o indeterminación del sexo.

¿Qué condiciones abrieron la posibilidad a la reforma de ley que cambia el registro del sexo en recién nacidos? ¿Cómo fue que la ambigüedad de genitales se relacionó con una nueva opción de registro? ¿Por qué la medida es rechazada justo por la población a la que pretende proteger? Avancemos en lo posible con estas primeras preguntas.

Desde 1990, ex pacientes de hospitales pediátricos que habían alcanzado la edad adulta comenzaron a denunciar que los protocolos médicos de atención a la intersexualidad con los que habían sido atendidos tuvieron resultados desafortunados (Chase, 2005). Con el tiempo, los problemas fueron quedando claros: las continuas revisiones de genitales realizadas durante años en el hospital pediátrico, propiciaron profundos sentimientos de vergüenza y una sensación de indefensión que se prolongaba y extendía a otras áreas en la vida adulta; las cirugías genitales que tenían por objetivo adaptar las formas y la funcionalidad del cuerpo 
a estándares preestablecidos acordes con el sexo asignado, causaron dolores permanentes e infecciones recurrentes en las áreas intervenidas; las secuelas de las cirugías realizadas en la infancia ataron a las personas al hospital; la asignación o reasignación de sexo recomendada por el equipo médico fue rechazada en algunos casos (Dreger, 2006).

El actual protocolo médico recomendado para atender los casos de intersexualidad fue establecido en Estados Unidos en la década de 1950. Fueron dos los grupos de especialistas dedicados a estudiar $\mathrm{y}$ atender los estados intersexuales. El primero se ubicaba en el Hospital Johns Hopkins de Baltimore y el segundo en la Gender Identity Research Clinic de la Universidad de California, en Los Ángeles. En el área de salud mental, el grupo del Hospital Johns Hopkins contaba con el psicólogo John Money y los psiquiatras Joan y John Hampson. El grupo de la Gender Identity Research Clinic, con el psicoanalista Robert Stoller. Money y Stoller conocieron recíprocamente su trabajo y, dado que partían de dos enfoques notablemente diferentes, no siempre coincidieron. En especial, Robert Stoller mostraba gran cautela ante las recomendaciones de John Money, que juzgaba aventuradas y excesivamente optimistas (Stoller, 1968).

Con el tiempo, el grupo ubicado en el Hospital Johns Hopkins ganó un gran reconocimiento. El nuevo paradigma modificó algunas medidas para la atención de los casos de intersexualidad: cambió la dirección del tratamiento que pasó de cirujanos y urólogos a endocrinólogos, estableció la toma de decisiones en grupo - lo cual indicaba la conformación de equipos médicos organizados bajo el modelo de clínicas de intersexo-y recomendó la participación de psiquiatras y psicólogos en el equipo. Esos cambios fueron vistos como avances (Gumbrach, Hughes y Conte, 2004).

No obstante, el cambio más radical del nuevo paradigma de atención fue que la intersexualidad comenzó a ser detectada a edades cada vez más tempranas. La ambigüedad de genitales en recién nacidos y bebés pasó a representar un signo de alarma que activaba al equipo de la clínica de intersexo (Kessler, 1998). Es conveniente recordar que en el siglo XVIII solo era posible explorar el interior de un cuerpo catalogado como hermafrodita mediante una autopsia (Foucault, 1985). Hacia 1950, la disciplina médica ya había incorporado en los hospitales el uso habitual de la tecnología que permitía explorar el interior del cuerpo e intervenir con procedimientos quirúrgicos a un ser humano vivo. El protocolo de atención a la intersexualidad incluyó la manipulación repetida del área genital de niñas y niños pequeños, así como las cirugías de genitales para alterar las formas originales y adaptarlas a los estándares predeterminados. Durante décadas esos procedimientos de alteración permanente a órganos y tejidos relacionados con la función reproductiva, así como otras intervenciones dirigidas a modificar las características sexuales atípicas, continuaron realizándose (Alcántara, 2012).

Cuando la intersexualidad comenzó a detectarse durante la infancia y la niñez, fue necesario decidir qué asignación sexual sería la más adecuada. En épocas anteriores una persona consolidaba su identidad como masculina, femenina o ambigua sin intervención de los médicos. Para decidir el sexo de asignación de un bebé o niño/a puesto en duda había que verificar su conformación 
orgánica y aclarar la edad máxima adecuada para reasignar el sexo. Para enfrentar los nuevos retos se desarrollaron nuevas estrategias. En la década de 1950 empezó a usarse el término "género" vinculado a la identidad. En los dos principales grupos pioneros de investigación y tratamiento ubicados en Estados Unidos, el "desarrollo" de la identidad comenzó a ser explicado a partir de la distinción y la oposición entre "sexo" y "género". Desde entonces, gender identity y gender role pasaron a formar parte de la terminología especializada. La intersexualidad y la transexualidad fueron los modelos ideales para investigar cómo se forma la identidad de género.

El término "sexo" quedó reservado para los componentes orgánicos, la materialidad corporal: apariencia y funcionalidad de los genitales, conformación de las gónadas y otros tejidos $\mathrm{u}$ órganos asociados con la reproducción. En sí mismo, el sexo ya tenía su complejidad, puesto que se estaba desarmando en múltiples elementos a los que, con el paso del tiempo, habrían de sumarse la estructuración genética y el metabolismo hormonal. El "sexo" fue definido como el conjunto de características biológicas naturales, los “elementos básicos irreductibles que ninguna cultura puede erradicar" (Money y Ehrhardt, 1972: 35).

El término "identidad de género" destacó el registro conductual y narrativo del sentido de pertenencia a un sexo. Sin embargo, no es posible contar con ese registro objetivable en el caso de bebés e infantes debido a su incipiente estado de maduración. El método empírico, que registra la experiencia a partir de los eventos observables, tuvo un acercamiento muy limitado a la complejidad de los procesos de sexuación. Un efecto producido por la aproximación metodológica empleada fue la idea de la "maleabilidad del género" antes de los dos años de vida. Después de esa edad, el progreso en el lenguaje, el tipo de juego y el avance en la socialización generan condiciones que permiten el registro de datos empíricos, los cuales - desde una mirada positivista - constatan el "establecimiento de la identidad de género". La noción de "rol de género" se desprendió de la noción de "identidad de género"; en la primera se resalta el registro del hacer y en la segunda el registro del decir. Por largo tiempo, este correlato conductual ha explicado el sentido de ser hombre o mujer con la lógica de una teoría de desarrollo infantil.

Durante la segunda mitad del siglo XX prevaleció la idea de que el tratamiento de los infantes intersexuales no traería mayores dificultades una vez impuesto. Se evaluaba la conformación corporal, las potencialidades a futuro y la situación en que se encontraba el desarrollo de la identidad de género; entonces se decidía el rumbo del tratamiento. Además de las medidas necesarias para preservar la salud del infante, se consideró que las intervenciones quirúrgicas eran indispensables. El número y tipo de intervenciones se decidía de acuerdo al caso en cuestión, pero en su mayor parte se indicaban biopsias, extirpación de gónadas y otros tejidos accesorios, y cirugías para remodelar los órganos genitales de acuerdo con la identidad asignada o reasignada. Aún hoy es frecuente encontrar la idea, entre la mayoría de los médicos especialistas, de que reducir la ambigüedad en las formas genitales para adaptar el cuerpo al género asignado, fija la identidad con mayor certeza. Desde un inicio, y en 
función de las posibilidades quirúrgicas, se prefirió la asignación femenina a la masculina dado que: "Es más factible reconstruir los genitales externos para crear una hembra funcional, particularmente cuando existe ya una vagina, que crear un pene masculino funcional" (Nelson, Mckay y Vaughan, 1980: 1425). Durante años, los cirujanos se han dedicado a mejorar las técnicas quirúrgicas - tan difíciles de aplicar en cuerpos tan diminutos-, maravillados al encontrar condiciones ideales de plasticidad. El tiempo ha mostrado que esos tratamientos, cuyo objetivo era atenuar el malestar de la diferencia corporal, instauraron la huella del estigma y ensombrecieron de manera radical la configuración subjetiva de las personas (Machado et al, 2015). En 2013 las intervenciones quirúrgicas practicadas en bebés y niños/as intersexuales fueron denunciadas ante la Comisión Interamericana de Derechos Humanos. En la actualidad existen documentos internacionales que identifican estos procedimientos como formas de abuso médico, entre ellos, los Principios de Yogyakarta (2007) y el último informe del relator especial sobre la tortura de la Organización de las Naciones Unidas (ONU) (OMS, 2014).

\section{"YO NENA, YO PRINCESA"}

El 9 de octubre de 2013 fue otorgado en Argentina un nuevo Documento Nacional de Identidad (DNI) a Lulú, quien para ese entonces tenía seis años de edad. Según la crónica de Mariana Carbajal (2014) publicada en la revista Debate Feminista, Lulú nació con genitales masculinos $\mathrm{y}$, al igual que su hermano mellizo, le fue asignado al nacimiento el sexo masculino. Su nombre era Manuel.
—Mi impresión era que tenía mellizos pero los dos tenían gustos opuestos - contó [Gabriela, la madre de Lulú] a esta cronista- A los 18 meses, cuando Manuel empezó a hablar, me decía: 'Yo nena, yo princesa.' Quería tener el cabello largo y para simularlo se ponía trapos en la cabeza; pedía que le compraran muñecas. Me pedía mis faldas, mi ropa, y se las quería poner. Yo pensé que era un juego - dice Gabriela, quien peregrinó por pediatras, neurólogos, psicólogos, buscando una respuesta. Un psicólogo me dijo que le faltaba presencia paterna, que le tenía que decir que era un nene, que le sacara la ropa de mujer. Fue un desastre. Mi hija vivía destrozada. Se escondía debajo de la cama, se ponía el cubre cestos del baño, que tenía holanes, como falda, y pasaba horas encerrada en el baño. Cuando le quitaba la ropa femenina, yo sentía que le arrancaba la piel. No te imaginas cómo lloraba. Podía llorar horas. El papá no lo podía tolerar. Decía: 'Yo no voy a tener un hijo puto.' Y escondía a Manuel cuando venían sus amigos. ¿Sabes con qué jugaba? Con un lápiz rosa. Hasta que vi un documental de National Geographic de una nena transgénero de Estados Unidos. Fue como si me pasara un camión por encima. Era la historia de mi hijo. Ahí entendí que era una nena trans, que su identidad era la de una nena. Lloré veinte días. Y reaccioné. Me dije: 'si quiere ser princesa, yo la voy a ayudar.' El complemento de ella siempre fue su hermano mellizo, que sabía lo que ella quería: si teníamos que comprarle un regalo y yo le preguntaba a él, me decía que a Manuel le gustaban las muñecas (Carbajal, 2014: 233).

Relata la madre que, a los cuatro años, su hijo le pidió que la llamaran con un nombre que eligió: "Yo no soy un nene. Soy una nena y me llamo Lulú" (Carbajal, 2014: 234). La madre de Lulú consultó a diversos especialistas. Al inicio el caso se trató con una terapia correctiva de reafirmación de género masculino: "había que reforzarle la 
masculinidad, había que obligarla a vivir en el género masculino, había que reprimirla y aplicar violencia de alguna manera, había que retarla, había que obligarla" (Video A, 2014). Después de haber visto el documental de National Geographic, la madre se sintió aliviada cuando llegó con una psicóloga de la comunidad homosexual en Argentina que le ratificó lo que pensaba: que su hija era una nena trans.

Al igual que en casos similares, la madre recibió un mar de críticas y descalificaciones tanto de personas que la conocían como de quienes no la conocían pero opinaban del caso en diferentes medios de comunicación. Para la madre y quienes la apoyaron, la petición del cambio de DNI y el reconocimiento jurídico de la existencia de Lulú ayudarían a proteger a la niña al facilitar su acceso a la educación y a la salud, y al promover un trato no discriminatorio. Carbajal describe en su crónica que el Registro de las Personas de la provincia de Buenos Aires se negó, en un inicio, a proporcionar un nuevo DNI; el argumento fue que la peticionaria —Lulú - tenía una edad inferior a los 14 años. Fue entonces que se tomó la decisión de difundir el caso en el diario Página/12 y que la madre escribió a la presidenta Cristina Fernández de Kirchner una carta para solicitar su ayuda en el procedimiento de cambio. Poco después, la Secretaría Nacional de Niñez, Adolescencia y Familia (Senaf), analizó y avaló la petición citando la Convención Internacional sobre los Derechos del Niño y la Convención Interamericana de Derechos Humanos.

El nuevo DNI registró la identidad percibida de Lulú, de acuerdo al género adoptado, mediante un cambio de nombre y sexo. El trámite fue realizado a partir de un procedimiento administrativo según la nueva Ley de Identidad de Género sancionada en Argentina en 2012. Ante las cámaras de televisión, la presidenta de Argentina entregó el nuevo DNI. La historia se convirtió en una noticia mundial, dado que por primera vez ocurría que un Estado apoyaba a una niña de seis años para tramitar el cambio de nombre y asignación de sexo en el Documento Nacional de Identidad.

La madre de Lulú relató lo ocurrido en una entrevista extensa que fue capturada en formato cinematográfico (Aramburú y Paván, 2014). También escribió un libro para dejar registro de lo sucedido, porque le interesa que la historia sea contada por ella, con las palabras de Lulú. En la presentación del libro, durante la $40^{\mathrm{a}}$ Feria internacional del libro en Buenos Aires, dijo: “en ese libro hay cuatro años de dolor, cuatro años de, quieran creerlo o no, la manifestación de una niña en el cuerpo de un niño. Loana [Lulú] es una niña trans, es una niña con genitales masculinos, que lucha todos los días para estar en una sociedad donde si no sos varón, no sos nena, no sos rosa, no sos celeste, no sabés dónde meterte" (Video B, 2014).

En internet se constata que hoy en día otros niños y niñas están viviendo circunstancias similares a la de Lulú, tanto en Argentina como en otros territorios, incluido México. Vivir en un rol de género distinto al señalado en los documentos de identidad conlleva grandes problemas desde una edad temprana; por ejemplo: para ingresar o mantenerse en la escuela, para recibir atención médica sin dificultades y para hacer efectivo que la identidad funcione a partir de documentos como el pasaporte. Ni hablar de las dificultades cotidianas 
y reiteradas, como el derecho a utilizar el baño público que se desea sin ser blanco de agresiones y violencia. No cabe duda que todos los niños y las niñas merecen vivir en un mundo que les respete y les ofrezca las mismas oportunidades de cuidado, amor y crecimiento sin importar su expresión de género.

Casos como el de Lulú también deberían reafirmar el compromiso ético de quienes somos consultados en la clínica y por las instituciones públicas para orientar respecto a los dilemas que se presentan en la actualidad: ¿qué hacer?, ¿cómo responder?, ¿conocemos con certeza cómo se configura y a qué edad se consolida la identidad de género?, ¿cuál sería la edad mínima para apoyar una solicitud de cambio de sexo registral?, ¿tenemos certeza de que en todos los casos la reasignación solicitada perdurará en un futuro? Sin dejar de colocar en primer término el interés de los niños y las niñas que viven esta difícil situación, es indispensable y urgente problematizar lo que está ocurriendo más allá del modelo médico de explicación que ofrece la transexualidad: ¿qué se revela y qué se esconde cuando leemos lo que acontece únicamente con los lentes de ese modelo? ¿Qué líneas en el tiempo y el espacio debemos reunir para comprender una situación tan compleja? Exploremos el panorama incorporando nuevos elementos.

La década de 1960 fue particularmente activa en la producción de diagnósticos mentales. En 1965 la Organización Mundial de la Salud (OMS) ${ }^{2}$

2 La OMS es el organismo especializado de las Naciones Unidas responsable de los problemas de salud y de la salud pública. Fue creada en 1948 con el objetivo de permitir a los profesionales sanitarios de los países miembros intercambiar conocimientos y experiencias que permitieran mejorar el nivel de salud. Una de sus tareas es la revisión de la Clasificación Estadística Internacional de las introdujo en la Clasificación Internacional de Enfermedades (CIE) el diagnóstico denominado transvestitism, un primer antecedente de lo que más tarde sería el grupo de diagnósticos referidos con el nombre gender identity disorders. El diagnóstico transsexualism aparece por primera vez en 1980, esta vez en la tercera edición del Manual de trastornos mentales y del comportamiento -DSM (Diagnostic and Statistical Manual of Mental Disorders), por sus siglas en inglés - publicado por la Asociación Psiquiátrica Americana (APA).

El diagnóstico de transexualidad fue el inicio de un cambio mayor que, en conjunto con otros factores, dio lugar a la creciente visibilidad de personas identificadas como transexuales quienes, con el tiempo, comenzaron a luchar para despatologizar su experiencia y validarla como una forma legítima de vida. Ese movimiento ayudó a reubicar la identidad de género en un lugar central y la colocó junto a otras características — percibidas como rasgos naturales - como la raza, el sexo y la edad:

Mientras $1 *$ s médic*s en Europa habían comenzado
a realizar cirugías de reasignación de sexo (CRS)
a principios de 1920, el transexualismo y las CRS
entraron en la imaginación popular general cuando
los medios de Estados Unidos informaron de manera
sensacionalista acerca del viaje de Christine Jorgensen
a Dinamarca, como alguien que había sido hombre y
había regresado a los EE UU en 1952 como una mujer

Enfermedades y Problemas Relacionados con la Salud (CIE). Entre otras funciones, la CIE permite recoger, difundir e intercambiar información estadística sanitaria para diseñar políticas públicas y desarrollar sistemas de atención en salud. La OMS tiene especial interés en estimular y llevar a cabo investigaciones sobre los criterios de clasificación y fiabilidad diagnóstica que aparecen en la CIE, así como para definir las categorías que emplea (OMS, 1999). 
trans con un nuevo cuerpo y un nuevo nombre $[\ldots]$ La publicidad en torno a la transición de Jorgensen eventualmente condujo a una mayor concientización popular, médica y psiquiátrica sobre los conceptos de identidad de género $y$, posteriormente, de género vivido [experienced gender], así como al reconocimiento de un número creciente de personas que deseaban cruzar desde el sexo que les asignaron al nacer a otro sexo. Las crecientes discusiones públicas sobre reasignación de sexo e identidad de género brindarían a aquell*s que eventualmente vendrían a identificarse como transexuales o transgéneros con un modelo, una categoría y un nombre para lo que sentían y deseaban. Con el tiempo aquello que alguna vez fue considerado una condición extremadamente rara, gradualmente se volvió más visible públicamente, y en años recientes, un número creciente de países, provincias y municipios han promulgado leyes civiles y de derechos humanos prohibiendo la discriminación basada en la identidad de género, en adición a otras características tales como la raza, la etnicidad, la edad, el sexo y la orientación sexual (Drescher, Cohen-Kettenis y Winter, 2012: 569). [La cita en español es retomada de la traducción no publicada de Mauro Cabral.]

La primera referencia de un diagnóstico asociado a la identidad de género en la infancia apareció en 1980 con la denominación Gender identity disorder of chilhood. El mismo diagnóstico se mantuvo en el DSM-III-R publicado en 1987, y cambia a Gender identity disorder in children para el DSM-IV en 1994 y DSM-IV-TR en 2000 (Drescher, Cohen-Kettenis y Winter, 2012). Finalmente, en el DSM-V, publicado en 2013, el diagnóstico aparece con el código 302.6, bajo el nombre Gender Dysphoria in Children. En la CIE, el diagnóstico aparece 10 años después, en 1990. Se encuentra en el Capítulo V denominado "Trastornos mentales y del comportamiento", catalogado como F64.2 Trastorno de Identidad sexual en la Infancia. El cuadro se describe como sigue:

[...] se caracteriza por un malestar intenso y persistente por el propio género junto al deseo (o insistencia) de pertenecer al sexo opuesto. Existe una preocupación constante con el vestido o las actividades del sexo opuesto, o un rechazo hacia el propio sexo. El diagnóstico requiere una profunda alteración en el sentimiento normal de masculinidad o feminidad. No es suficiente la simple masculinización de los hábitos en las chicas o el afeminamiento en los chicos (OMS, 1999: F64.2).

Las características diagnósticas se presentan por separado para mujeres y para varones; se indica que los síntomas deben aparecer antes de la pubertad y estar presentes durante al menos seis meses. Para evaluar la identidad de género se atiende a la dimensión conductual que verifica la presencia de evidencia enlistada en dos registros: el decir y el hacer. Sobre el registro del decir, para las niñas se considera la afirmación de que ella tiene, o que le crecerá, un pene y la afirmación de que no quiere que le crezcan los pechos ni tener la menstruación; para los niños, la creencia en que él crecerá hasta convertirse en mujer (no solamente en lo referente al papel de ésta), la creencia en que su pene y sus testículos son molestos o que desaparecerán y la creencia de que sería mejor no tener testículos ni pene. En el registro del hacer se consigna en el caso de las niñas un rechazo marcado y persistente hacia los atuendos femeninos habituales, la insistencia de vestir ropas típicamente masculinas (ropa interior y otros accesorios de los chicos, por ejemplo), el 
repudio persistente de las estructuras anatómicas femeninas y la negativa a orinar sentada; en el caso de los niños la preocupación por actividades típicamente femeninas, la preferencia por vestir o simular atuendos femeninos, el deseo intenso de participar en los juegos y pasatiempos de las chicas, el rechazo de los juguetes, juegos y actividades típicamente masculinas. Por supuesto, dividir las conductas en apropiadamente femeninas o masculinas para marcar las fronteras de salud entraña su patologización. Los estudios de antropología feminista, en particular Margaret Mead (1994 [1949]), mostraron evidencia clara de que la clasificación masculino/femenino depende de factores culturales, geográficos y temporales. Esto quiere decir que ni lo masculino ni lo femenino contienen rasgos fijos, permanentes o naturales.

En la actualidad la OMS está preparando la $11^{\mathrm{a}}$ edición de la CIE. Para la revisión de los diagnósticos que aluden a la identidad de género, se ha conformado un grupo de trabajo específico que evaluó los datos clínicos y de investigación que sobre el tema existen desde 1992 (Drescher, Cohen-Kettenis y Winter, 2012). Los cuestionamientos principales en torno al grupo de diagnósticos referentes a la incongruencia de género se refieren a la necesidad y el impacto que pueden tener tales categorías en la CIE. No parece definitiva aún la decisión de clasificar ese grupo de diagnósticos como trastornos mentales. Se ha dicho que excluir los diagnósticos contribuiría a disminuir la estigmatización de las personas trans, pero al mismo tiempo se argumenta que obstaculizaría su atención en los centros de salud y el pago de las intervenciones médicas a cargo de aseguradoras y sistemas de salud estatales.
Son notables la gran sensibilidad y la preparación del grupo de trabajo de la OMS que emitió sus primeras recomendaciones sobre qué hacer con los diagnósticos referidos a la identidad de género para la CIE 11; sin embargo, aún está pendiente una revisión más cuidadosa de lo que sucede cuando un diagnóstico de esta índole es aplicado a la infancia. No podemos asumir de manera inmediata que exista una línea recta que conecte en un proceso continuo y generalizable los tres momentos señalados por los diagnósticos relativos al trastorno de identidad sexual (CIE) o disforia de género (DSM): infancia, adolescencia y edad adulta. En la dinámica de subjetivación que tiene lugar en la infancia, en la adolescencia y en la edad adulta participan muy diversos elementos que se articulan en forma singular e impredecible. A esto debemos agregar que las relaciones de poder y jerarquía configuran una escena diferente cuando se trata de niños y niñas. Especialmente, cuando la asignación del diagnóstico depende de la ideología y de la implicación política del clínico consultado, como lo han señalado con claridad Alice Dreger (2009) y Bernadette Wren (2014).

Marta Lamas (2012) refiere que en 1973 el impulso de las batallas político-culturales de los movimientos feminista y gay cambió el estatuto de la homosexualidad, primero en Estados Unidos y luego en Europa. La APA tomó la decisión de sacar el diagnóstico de homosexualidad de la tercera edición del DSM. Para Lamas, "lo paradójico es que la despatologización de la homosexualidad, que introduce un cambio radial en la concepción jurídica de los derechos ciudadanos, se da casi al mismo tiempo que se instala la patologización de 
las expresiones no normativas del género" (Lamas, 2012: 227). La exclusión de la homosexualidad como trastorno mental en el DSM se acompañó de la inclusión de los trastornos de identidad de género, entre ellos el Gender identity disorder of chilhood. Esto contribuyó a incrementar la rigidez con que los clínicos evalúan el género en la infancia. Los estereotipos sociales -inmersos en los criterios diagnósticos de los trastornos de identidad - anticipan una identidad donde ocurre una exploración. Armar la identidad sexuada requiere del juego, de la experimentación, de la interpelación, de la incorporación al mundo. La producción subjetiva vinculada a la diferencia sexual avanza lenta e impredecible, y excede el correlato conductual y el registro del decir.

Wilson, Griffinn y Wren (2002) indicaron que la mayoría de los estudios de seguimiento realizados con niños diagnosticados con gender identity disorder reportaban una identidad homosexual en la edad adulta, por lo tanto - argumentaron-, más que ser la indicación de un desorden, la conducta de género variable puede estar indicando el desarrollo normal de un futuro patrón homosexual. Dada la diversidad de factores y dimensiones que participan en la estructuración psíquica de la diferencia sexual no puede existir un método para determinar qué niños diagnosticados en la infancia persistirán y asumirán una identidad trans en la edad adulta y quiénes no lo harán.

El tema del diagnóstico aplicado a la niñez ha sido analizado por el grupo de expertos convocado por Global Action for Trans Equality (GATE). Dicho grupo presentó un análisis detallado sobre la categoría Incongruencia de género en la infancia y declaró su preocupación por el hecho de que aparezca en la próxima versión de la CIE. El grupo encuentra que:

En primer lugar, no hay un consenso claro entre los investigadores y profesionales de la salud en relación con la necesidad o la aplicabilidad global de tal diagnóstico. En segundo lugar, la variación de género en la infancia no requiere ninguna intervención médica tal como la terapia hormonal o procedimientos quirúrgicos. Más bien, los niños necesitan información y apoyo en la exploración de su identidad y expresión de género, y deben hacer frente a entornos socioculturales que con frecuencia son hostiles a la variación de género. En tercer lugar, adjuntar un diagnóstico médico a la diversidad de género en la infancia contradice el compromiso de la OMS para respetar, más que patologizar, la diversidad sexual. En concreto, la investigación indica que es imposible distinguir confiablemente entre un niño género-variante que crecerá hasta convertirse en trans y un niño génerovariante que llegará a ser gay, lesbiana o bisexual, pero no trans. Como tal, al conjuntar la variación de género y la orientación sexual, la categoría GIC [Gender Incongruence of Childhood] abona a una repatologización de la homosexualidad (GATE, 2013: 5). ${ }^{3}$

Al respecto, GATE (2013) presentó varias propuestas alternativas para que en la CIE se registren estos casos con fines estadísticos sin adjudicar un diagnóstico que defina como trastorno la variabilidad en la expresión de género en la infancia.

En México, se encuentra en curso un estudio de campo cuyos resultados preliminares muestran una problemática muy similar a la señalada (Alcántara, 2014). La asignación de un diagnóstico como trastorno de identidad sexual, disforia de

3 La traducción del original en inglés es mía. 
identidad de género, una etiqueta de niño o niña trans o ninguno de los anteriores, tiene una relación directa con la orientación teórica que adscriba el clínico consultado, con sus creencias personales y con su implicación política. Además de esto, se identificaron tres grupos de factores que están relacionados con la asunción o no del diagnóstico. Primero, importa mucho cómo la familia del niño o la niña - en especial, la madre - significan lo que sucede, cómo se articula el diagnóstico con la dinámica familiar, cómo se reconfiguran las relaciones de la familia y qué redes sociales se generan de la situación en curso. Segundo, son determinantes las circunstancias singulares del contexto local en que se presenta el caso: ¿existe una legislación amigable para resolver el cambio de identidad jurídica?, ¿qué tan solidaria es la comunidad LGBT local?, ¿qué presencia tiene el tema de infancia trans en los medios de comunicación disponibles? Tercero, la edad del niño o la niña en cuestión es un asunto importante; mientras el infante tenga una edad menor, todos esos factores tendrán un peso mayor al momento de definir la situación. Esto ocurre porque en la infancia los procesos de autonomía están sujetos al transcurso de la maduración biológica y a las oportunidades que brinda el entorno para su desarrollo.

El acceso de niños y niñas a servicios de salud, a inscribirse y a permanecer en la escuela, así como a contar con documentos de identidad que avalen su género vivido no puede sujetarse a cubrir criterios de clasificaciones y modelos psicopatológicos. Es fundamental garantizar el derecho al cambio de sexo registral en los documentos de identidad y comprender que la solicitud no implica que en un futuro esa decisión se mantendrá. Los retos que como sociedad enfrentamos son nuevos y requieren soluciones creativas que permitan reformular las prácticas jurídicas. En las legislaciones más avanzadas existe la tendencia a fundamentar la resolución de los casos expuestos en una argumentación apoyada en los principios de derechos humanos y los derechos de niños y niñas (Regueiro, 2012; Cabral, 2014). Atender fundamentalmente los nuevos acomodos de construcción de género desde la perspectiva de los derechos humanos evita adscribir criterios esencialistas que parten de una visión medicalizada, promueve un alejamiento de la victimización y abona a la afirmación de la agency individual (Lamas, 2012). Las transformaciones sociales abren la posibilidad a otros lugares legítimos de existencia, pero esto es un proceso lento y gradual.

Padres y madres de familia, y quienes tienen a su cargo a niños y niñas también deben tomar con cautela interpretaciones que anticipan bajo esquemas preconcebidos algo que está aún por acontecer (Dreger, 2009). La identidad de género es la capa superficial - evidente-de procesos complejos que no son voluntarios, ni controlables, ni predecibles. La construcción de la identidad de género comienza con la expectativa manifiesta $\mathrm{y}$ los deseos inconscientes de los padres, continúa lentamente y atraviesa varios momentos críticos. Entre otros momentos, por mencionar algunos, se pueden destacar: la experimentación erógena del cuerpo, la asignación sexual al nacimiento, la designación del nombre, la incorporación al lenguaje, el descubrimiento de la diferencia genital, la asimilación de los cambios puberales que se presenten, la práctica sexual, la exploración del 
deseo, la exploración vinculada con la potencialidad reproductiva, la configuración deseante respecto a la reproducción, el ser padre o el ser madre, las experiencias de vida referentes a las parejas. No hay una experiencia de vida predeterminada, la configuración subjetiva y deseante no termina más que con la muerte.

\section{GENDER FLUID Y LA BÚSQUEDA DE LO QUEER} En mayo de 2011, el diario The Toronto Star publicó un reportaje sobre una pareja canadiense (Kathy Witterick y David Stocker) que había decidido criar a su bebé manteniendo en secreto el sexo asignado al nacimiento (Poisson, 2011). El objetivo de la pareja era educar a su bebé con libertad de elección y confrontar los límites que impone el género binario. También basaron su decisión en la experiencia de crianza de Jazz y Kio, sus hijos mayores, en ese entonces de cinco y dos años de edad. A su hijo mayor, Jazz, le gustaba usar vestidos y también el color rosa, y optó por traer el pelo largo peinado con trenzas. A decir de la madre, las preguntas que Jazz formulaba sobre el género fueron en parte lo que inspiró la decisión de la pareja respecto a dejar en suspenso, de manera pública, la asignación sexual de Storm (Witterick, 2013).

La noticia se difundió a través de los medios de comunicación impresos y electrónicos. El caso desató una gran controversia global. Hubo quien calificó la situación como un experimento social. Las opiniones estaban divididas: algunas personas apoyaron e incluso celebraron la decisión de los progenitores; otras no. Los argumentos a favor aprobaban una educación fuera de los estereotipos de género y las limitaciones que éstos imponen. Los argumentos en contra reclamaban el derecho de es infante a ser educad_en una identidad común, libre de estigma y discriminación. También se cuestionó el límite en las decisiones que los progenitores pueden tomar al momento de decidir cómo educar a sus hijos.

Las nuevas prácticas de crianza que se anuncian como desafiantes al binarismo de género son conocidas como gender fluid. En la introducción al libro Chasing Rainbows: Exploring Gender Fluid Parenting Practices (2013), sus editoras Fiona Joy Green y May Friedman explican que:

[gender fluid parenting practices significa que] para ayudar a los niños a expresar su género de manera que se sientan cómodos consigo mismos y que no se ajusten a roles de género estrictos y a expresiones socialmente prescritas, muchos padres intentan alterar las fronteras y los binarios de género. Ellos acompañan la creatividad de género de sus hijos para apoyarles en múltiples oportunidades para desarrollar y practicar su expresión [de género] (Friedman y Joy, 2013: Introducción, versión para Kindle). ${ }^{4}$

A decir de las editoras, las experiencias que aparecen en el libro se suman a otras que se encuentran explorando y experimentando la gender fluidity. $\mathrm{La}$ autoras relatan que otros ejemplos inspiradores son el de Thomas Beatie — quien fue anunciado en los medios de comunicación bajo el encabezado del primer hombre embarazado- o el libro Gender Outlaws: The Next Generation, de Kate Bornstein y Bear Bergman, y una miríada de blogs que muestran cada vez con mayor fuerza que el género es más bien un continuo que un binario. Además de Storm,

$4 \mathrm{La}$ traducción del original en inglés de las citas de este texto, es mía. 
se sabe que otros niños y niñas habitantes de países anglófonos están siendo criados bajo este esquema, también conocido como género neutro.

La madre de Storm, Kathy Witterick, escribió un capítulo para el libro editado por Friedman y Joy (2013). En él escribe lo siguiente:

El género es literalmente un conjunto de reglas arbitrarias dispuestas por los adultos dominantes con el fin de mantener los diferenciales de poder [...] La inconformidad de género no es un problema a solucionar [...] El género forma gran parte del mundo real. Es un lugar socialmente significativo donde los niños tienen el derecho de expresar su verdadero ser, en un camino de estimación y responsabilidad. Para proteger su agency, los niños necesitan practicar para cuestionar todos los días los límites de su expresión, límites que están en todas partes. Comprar unas botas de lluvia en un mundo rosa o azul, Dora o Diego, héroes de acción o princesas; todo esto nos puede conducir a reflexionar acerca de los estrangulamientos capitalistas que restringen la libertad de los niños para expresar su forma de ser única (Witterick, 2013: capítulo 1, versión para Kindle).

En la introducción al libro Chasing Rainbows, Fiona Joy Green y May Friedman comentan que la historia de Storm inspiró el proyecto. La decisión de la pareja Witterick y Stocker de no revelar el sexo de su tercer hijo fue el detonador que les llevó a preguntarse cómo se puede promover la creatividad y la libre determinación de las criaturas en un mundo organizado en estereotipos de género. En el mismo libro se relatan otras experiencias personales que ensayan la crianza fuera del modelo de la conformidad de género. Las autoras de esos textos describen instantes de alegría, desafíos, situaciones de tensión y confrontación que día a día viven ellas y sus familias. Las prácticas de crianza que ven como algo posible la autodeterminación del género en la niñez mezclan su argumentación con un tono emotivo y seductor: los padres y las madres que están comprometidos con la libertad de elección sienten un amor incondicional por sus hijas e hijos.

Casi todos los autores y las autoras que participan en el libro Chasing Rainbows viven en Canadá. Al igual que la madre de Storm, se trata de académicos y activistas que a la vez son madres o padres de familia. El libro aborda experiencias de la vida privada que no obstante tienen un gran impacto político. Witterick relata que su tercer hijo, Storm, nació dos semanas después de que ella completó su doctorado en estudios de la mujer. Fiona Joy Green menciona en la introducción de Chasing Rainbows (2013) que la familiaridad con el tema de la autodeterminación de género de los/ as niños/as partió de la experiencia con su hijo veinticinco años atrás, quien desde preescolar "conscientemente exploraba y practicaba su género"; la autora relata que ella misma y su familia vivieron esas experiencias en forma aislada y sintieron que otros miembros de su familia, amigos y conocidos los calificaron como una anomalía. Hoy en día, afirma que ella y su familia forman parte de una comunidad global emergente construida a partir de redes de filiación y amistad, y conectada a través de internet, redes sociales y otros medios. Fiona enseña estudios de género y de la mujer.

Quienes defienden la crianza bajo el esquema del gender fluid se declaran con frecuencia feministas, partidarias de la teoría académica, particularmente 
de autoras feministas catalogadas como queer: en el libro se menciona a Judith Butler, Judith Halberstam y Kate Bornstein, entre otras. No es una operación sencilla cuando la teoría académica se emplea como la guía de nuevas prácticas de crianza. La categoría género, cuya producción académica es vasta y heterogénea, es retomada con el interés de guiar la transformación de los procesos de producción de identidades. El género se resignifica en actos de la vida cotidiana y es leído como un instrumento de vigilancia y de control cuya estructura presiona por restablecer relaciones artificiales de concordancia. De esta lectura parece derivar la hipótesis de que si el género se produce, entonces es posible desarticular el binarismo interviniendo las prácticas sociales y corporales, los actos que performan el género.

Desde esta perspectiva, el género también es descrito como un juego que se puede explorar de maneras creativas. Se trataría de elegir la identidad desde una edad muy temprana retando el binarismo de género que impone una socialización tradicional. Aquí es importante atender algunas preguntas: ¿en qué medida es posible la autodeterminación de un infante?, ¿qué restricciones impone la edad a la libertad de elección? Dada la naturaleza de la construcción subjetiva - socialización, configuración deseante, procesos inconscientes, resonancia emocional-, ¿en qué medida es posible que operen preferencias individuales en momentos de exploración y búsqueda?

En las prácticas de crianza que experimenta la gender fluidity confluyen el activismo y el pensamiento teórico. Marta Lamas explica que en Norteamérica, especialmente en los Estados Unidos, se ha desarrollado de manera consistente un pensamiento teórico y una comunidad en torno al concepto transgender que reúne en alianza política a un conjunto de prácticas críticas dirigidas a develar la opresión del binarismo y a mostrar un abierto rechazo a las normas de género tradicionales. En la década de 1990, explica, la transgeneridad:

\begin{abstract}
ya no se veía como una patología sino como una posibilidad existencial liberadora de una sociedad con rígidos estereotipos [...] se empezó a entender el fenómeno transgénero como un producto de la diversidad humana $[\ldots]$ muchos se reconocieron en esa sensibilidad que cuestiona radicalmente las normas heterosexistas de la cultura occidental. Esto produjo un cambio sustantivo en el clima emocional de una naciente comunidad: del miedo o la vergüenza se pasó a la exigencia de igualdad política y justicia social (Lamas, 2012: 222).
\end{abstract}

Lamas (2012) refiere que a partir de 2000 el término transgender se presenta consistentemente en medios masivos de comunicación, se traslada vía internet y redes académicas, y se generaliza dado el fenómeno de americanización. ${ }^{5}$ La transgeneridad empuja a abrir nuevos espacios en el imaginario social al explorar otras vías para acceder a una vida legítima, digna y con garantía de igualdad de derechos, fuera de los esquemas heteronormativos

5 Marta Lamas retoma el término americanización - acuñado por Bolívar Echeverría - para explicar la globalización del modelo medicalizado con el cual se atiende hoy día la transexualidad. Dicho modelo incorporó las técnicas de cirugía reconstructiva en el tratamiento, se presentó como un progreso producto de la modernidad y se insertó en los mecanismos del mercado de consumo. Bolívar Echeverría sostiene que el conjunto de la vida económica, social y política en el último medio siglo se encuentra permeado por la versión dominante del progreso americano definido por Estados Unidos bajo el paradigma del desarrollo (Bolívar Echeverría citado en Lamas, 2012: 9). 
y sexistas de género. Gradualmente estas transformaciones van produciendo cambios en los sistemas jurídicos, los cuales ayudan a construir un piso común de igualdad ciudadana (Lamas, 2014). Esta tendencia va en aumento pues cada año incrementa el número de países que permiten el reconocimiento jurídico de los cambios de sexo y experimentan otras formas de registro del sexo que escapan al esquema binario hombre/mujer.

Lamas (2014) advierte que abordar la dimensión social y política de las identidades es tan importante como comprender la dimensión psíquica del proceso de construcción de la identidad. A lo anterior agregaría yo que para llevar a cabo esta tarea es indispensable entender las distintas formas en que se conciben teóricamente el sexo y el género, las relaciones que entre ellos se establecen y los desplazamientos y traslapes que ocurren cuando se utilizan las nociones identidad sexual e identidad de género (Alcántara, 2013). También es indispensable preguntarse qué aspectos quedan fuera cuando abordamos los temas aquí expuestos desde el paradigma de la identidad, y las categorías de sexo y de género. Es fundamental no perder de vista que: 1) "no se puede no hacer género: es inevitable hacer género, aunque a veces se hagan transgresiones" (Lamas, 2014: 148); 2) la dimensión de lo sexual abarca algo más que sexo y género (de Lauretis, 2011); 3) "las identidades sexuales y de género no se construyen voluntariamente, sino que están cruzadas por procesos psíquicos inconscientes" (Lamas, 2014: 174), 4) la inscripción y representación de la diferencia sexual ocurren de manera simultánea en múltiples dimensiones: el orden social, la cultura, el orden simbólico, el lenguaje, la dimensión imaginaria, el campo psíquico, la dimensión biológica, el cuerpo (Alcántara, 2012).

\section{UN ESBOZO DE TEMAS PENDIENTES}

En Queer Texts, Bad Habits and the Issue of a Future, Teresa de Lauretis (2011) advierte que en gran parte del mundo occidental, el énfasis en los términos empleados para designar a las identidades sexuales no normativas - LGBTI e incluso el término queer - ha provocado que se privilegie el género sobre la sexualidad, es decir, el vínculo social sobre lo propiamente sexual. De Lauretis es capaz de reunir las complejidades del pensamiento feminista posestructuralista con una lectura detallada que retorna a los fundamentos del psicoanálisis freudiano. Desde esta mirada, subraya que la teoría queer adolece de una falta de conexión entre sexualidad y género. $\mathrm{Su}$ señalamiento identifica con claridad que esta situación deriva del repetido desencuentro entre feministas y psicoanalistas. Desde el feminismo se suele desdeñar el pensamiento de Freud sobre la sexualidad, acusando al psicoanálisis de ser una teoría patriarcal y falocéntrica. Por su parte, el psicoanálisis disminuye la importancia de la teoría feminista y desestima sus categorías analíticas catalogándolas de producciones sociológicas que poco tienen que decir acerca de la dimensión inconsciente. Incluso, dice la autora, las feministas lacanianas evitan el tema del género "como una cuestión de principios, ya que en el psicoanálisis lacaniano es de hecho un nonissue" (de Lauretis, 2011: 252). ${ }^{6}$

6 La traducción del original en inglés de las citas de este texto, es mía. 
Por ello es relevante el análisis que de Lauretis realiza sobre la sexuación, el papel que el género juega en dichos procesos, lo que implica la dimensión sexual en la vida del ser humano, la diferencia entre sexualidad y género, entre teoría y política.

De entrada, de Lauretis nos recuerda que un análisis de la sexualidad no puede restringirse a los procesos reproductivos, a los eventos genitales, al comportamiento sexual o a los mensajes conscientes del binarismo de género. La sexualidad se inscribe como algo más que sexo, la aportación fundamental del psicoanálisis es atender la sexualidad como un exceso inmanejable del afecto, "un enigma sin solución y un trauma sin resolución", una dinámica energética, una inscripción heterotópica que "en secreto socava el lenguaje". De Lauretis nos invita a considerar la sexualidad en el sentido freudiano, a partir de la dinámica pulsional, su carácter obstinado y destructivo que se refleja en las dificultades y malestares que en torno a ella se producen tanto en el sujeto como en lo social. Teresa de Lauretis, considerada una de las fundadoras más relevantes de la teoría queer, señala:

Si vamos a reclamar lo queer en su significado sexual contestatario, y como verdaderamente incluyente de lo sexual, necesitamos una concepción de la sexualidad que vaya más allá de los equívocos nebulosos del género, así como de las preocupaciones médicas relacionadas con la funcionalidad reproductiva. Yo sugiero que tengamos una concepción como la que Freud teorizó, una sexualidad de pulsiones parciales y que muestra más claramente sus manifestaciones despejadas en la infancia: una sexualidad polimorfa, no reproductiva, buscadora de placer, compulsiva y rebelde (de Lauretis, 2011: 248-249).
En su análisis, de Lauretis utiliza su amplio conocimiento del psicoanálisis freudiano y además retoma las elaboraciones que Jean Laplanche introdujo a la cuestión del género y la sexualidad (Laplanche 2007, citado en de Lauretis, 2011). Laplanche parte de que:

[...] la sexualidad no es innata ni está presente en el cuerpo al nacer, sino que viene del otro adulto y es un efecto de seducción. Se implanta en el recién nacido -al principio sin lenguaje (como la etimología de la palabra infants implica) e inicialmente sin egopor las necesarias acciones del cuidado materno, alimentación, limpieza, mantenimiento, y más, a través de mensajes enigmáticos que se transmiten, enigmáticos, no solo porque el bebé no es capaz de traducirlos, sino también porque están imbuidos con las fantasías sexuales (in)conscientes de los adulto(s), progenitor(es) o cuidador(es). Intraducibles, estos significantes enigmáticos son sujetos a la represión primaria y constituyen el primer núcleo del inconsciente del infante, el inconsciente primigenio. Traducciones parciales ocurren cuando el niño crece y se forma y desarrolla el ego, pero ello, también, deja residuos sin traducir que habitan en el aparato psíquico del individuo como el sin recuerdo de una memoria de excitaciones corporales y placeres. Dicha memoria inconsciente marca la acción, en palabras de Laplanche, "como una astilla en la piel", o nosotros podríamos decir, como un virus instalado en una computadora. Eso permanece vivo, aunque indetectable, $y$ es reactivado en la sexualidad adulta, a veces en formas que nosotros encontramos vergonzosas o inaceptables. De ahí vienen los conflictos, ya sean morales o neuróticos, que todos experimentamos en la vida sexual (de Lauretis, 2011: 250-251).

Mientras que la sexualidad es un implante somático producto de excitaciones psicofísicas, 
el género no se implanta en el cuerpo físico, sino que es enviado y recibido a nivel consciente o preconsciente durante la formación del Yo. El género es una construcción social y normativa, binaria, que se asigna - social y legalmenteen función de cómo los adultos interpretan las formas genitales. Es por ello que modificar las formas de sociabilidad que instauran la identidad de género no implica transformar en el mismo sentido la dinámica psíquica. Las formas genitales no definen de antemano el camino de sexuación de un sujeto, pero sin duda la materialidad corporal y las posibilidades específicas de cada cuerpo incluida la potencialidad reproductiva - , marcan de manera importante la configuración subjetiva.

Según Laplanche, el desplazamiento conceptual de la identidad sexual a la identidad de género, en los discursos actuales, enfatiza la cancelación de la sexualidad infantil a favor de una categoría más aceptable para la autocomprensión del adulto (Laplanche 2007, citado en de Lauretis, 2011). El género borra o rechaza la dimensión inconsciente de la sexualidad infantil, cuyo despliegue es caracterizado por Freud como perverso y polimorfo.

El problema del género es la torcedura en el sexo: lo perverso, lo infantil, la vergüenza, el asco, lo "enfermo", los aspectos destructivos y autodestructivos de la sexualidad que la identidad personal rara vez confiesa y el discurso político sobre el género puede eludir o negar por completo. Para el tema de género, como las demandas por el reconocimiento legal de las nuevas o cambiantes identidades de género lo demuestran, es uno mismo quien requiere la aceptación y la validación social (de Lauretis, 2011: 253).
Transformar la estructura de género es el objetivo político de más de un movimiento social. No debe olvidarse que la diferencia sexual se inscribe no sólo en el orden social, en las formas culturales o en las posibilidades jurídicas. Esto no quiere decir que se desaliente la persecución de una utopía de igualdad, sino que para alcanzar esa meta debemos reconocer que las operaciones psíquicas de estructuración subjetiva ocurren más allá de la conciencia, la voluntad y la individualidad. En contraste con lo que dicta el sentido común, no es sólo el infante quien participa en los procesos de subjetivación que le llevarán a tomar un lugar en el mundo. Más allá del actual paradigma que restringe nuestra comprensión, la producción de la identidad sexuada comienza aún antes del nacimiento y no se termina sino con la muerte. El sentimiento que un niño o niña tiene de su lugar en el mundo está ligado con la forma en que su vida cuenta o no para alguien más, y cuenta para alguien sin haber tenido por ello que ausentarse de sí.

Para de Lauretis (2011) lo queer es más una teoría, que un programa de acción política; esto no quiere decir que no pueda existir una política queer, sino que se necesita una especie de traducción de una a la otra, de lo figurativo a lo referencial, de las palabras a las cosas.

El panorama presentado es complejo y anuncia un horizonte por venir que aún no es claro. Se consideró imprescindible delinear una serie de elementos para ser analizados desde diversos ángulos. Como un hypomnémata, en el artículo se privilegió la escritura de lo ya dicho: "reunir lo que se ha podido oír o leer, y con un fin que es nada menos que la constitución de sí" (Foucault, 2010 
[1994]: 941). Al igual que un caleidoscopio que podemos girar para producir diferentes figuras, las formas y los colores mostrados cambiarán según el haz de luz pueda descomponer la enorme cantidad de elementos que se condensan y entrecruzan en múltiples dimensiones. Ésta es una tarea apremiante que recién iniciamos. ¿

\section{AGRADECIMIENTOS}

Aunque asumo la responsabilidad de lo aquí expuesto, las ideas presentadas en este texto se han beneficiado de muchos intercambios mantenidos durante años. En especial agradezco a Ana Amuchástegui, Marta Lamas, Rodrigo Parrini, Hortensia Moreno y Benjamín Mayer por las conversaciones, la paciencia, la generosidad y la amistad. 


\section{BIBLIOGRAFÍA}

Alcántara, Eva (2012), "Llamado intersexual. Discursos, prácticas y sujetos en México”. México, Universidad Autónoma Metropolitana, tesis de doctorado en Ciencias Sociales.

Alcántara, Eva (2013), “Identidad sexual/rol de género”, en Debate Feminista, año 24, vol. 47, pp. $172-201$.

Alcántara, Eva (2014), "Prácticas, discursos y procesos de subjetivación vinculados a las regulaciones médicas y jurídicas del sexo-género en la infancia”. México, Universidad Autónoma Metropolitana, Departamento de Educación y Comunicación, Reporte anual de investigación, diciembre.

Aramburú, María y Valeria Paván (dir.) (2014), Yo nena, yo princesa, entrevista con Mónica Gabriela Mancilla en formato de cine, proyectada en México durante la XXVII Conferencia Mundial de ILGA, Argentina.

Ariès, Philippe (1986), “La infancia”, en Revista de Educación, núm. 281, pp. 5-17.

Bustelo, Eduardo (2011), El recreo de la infancia: argumentos para otro comienzo. Argentina, Siglo XXI.

Cabral, Mauro (2014), "Derecho a la igualdad: Tercera posición en materia de género. Corte Suprema, Australia, NSW Registrar of Births, Deaths and Marriages v. Norrie, 2 de abril de 2014", en Revista Derechos Humanos, año III, núm. 8, consultado el 20 de mayo de 2015, pp. 199-212. URL: http:// www.infojus.gob.ar/cabral-mauro-derecho-igualdad-tercera-posicion-materia-genero-corte-suprema-australiansw-registrar-of-births-deaths-and-marriages-norrie-2-abril-2014-dacf150277-2014-12/123456789-0abcdefg7720-51 fcanirtcod.

Carbajal, Mariana (2014), "Lulú”, en Debate Feminista, año 25, vol. 49, pp. 231-240.

Chase, Cheryl (2005), "Hermafroditas con actitud: cartografiando la emergencia del activismo político intersexual”, en Carmen Romero Bachiller, Silvia García Dauder y Carlos Bargueiras Martínez (Grupo de trabajo Queer) (eds.), El eje del mal es heterosexual. Figuraciones, movimientos y prácticas feministas queer. Madrid, Traficantes de sueños, pp. 87-111. 
DB (Deutscher Bundestag) (2013), Dokumentations und Informationssystem, Deutschen neues Intersex Gesetz Neues Intersex-Gesetz $\$ 22$ (3) PStG, consultado el 22 de agosto de 2014, URL: http://dipbt.bundestag.de/extrakt/ba/WP17/451/45180.html.

De Lauretis, Teresa (2011), "Queer Texts, Bad Habits, and the Issue of a Future”, en GLQ, vol. 17, núm. 2-3, pp. 243-263.

Dreger, Alice (2006), "Intersex and Human Rights: The Long View”, en Sharon E. Sytsma (ed.), Ethics and Intersex. Dordrecht, Springer, pp. 73-86.

Dreger, Alice (2009), "Gender Identity Disorder in Childhood: Inconclusive Advice to Parents”, en The Hastings Center Report, vol. 39, núm.1, pp. 26-29.

Drescher, Jack, Peggy Cohen-Kettenis y Sam Winter (2012), "Minding the body: Situating gender identity diagnoses in the ICD-11”, en International Review of Psyquiatry, vol. 24, núm. 6, pp. 568-577.

Foucault, Michel (2000 [1976]), Historia de la sexualidad 1. México, Siglo XXI.

Foucault, Michel (1985), Herculine Barbin, llamada Alexina B. España, Revolución.

Foucault, Michel (2010 [1994]), "La escritura de sí”, en Obras esenciales. Barcelona, Paidós.

GATE (Global Action for Trans Equality) (2013), "Critique and Alternative Proposal to the 'Gender Incongruence of Childhood' Category in ICD-11", consultado el 4 de febrero de 2015, URL: https:// globaltransaction.files.wordpress.com/2012/03/critique-and-alternative-proposal-to-the-_gender-incongruenceof-childhood_-category-in-icd-11.pdf.

Grupo de expertos en derechos humanos (2007), Principios de Yogyakarta, consultado el 21 de marzo de 2008, URL: http://www.yogyakartaprinciples.org/principles_sp.pdf.

Gumbrach, Melvin, Ieuan Hughes y Felix Conte (2004), “Trastornos de la diferenciación sexual”, en Reed Larsen, Henry Kronenberg, Shlomo Melmed y Kenneth Polonsky (coords.), Williams Tratado de Endocrinología. Madrid, Elsevier, pp. 917-1086. 
Joy, Fiona y May Friedman (eds.) (2013), Chasing Rainbows: Exploring Gender Fluid Parenting Practices. Canada, Demeter Press, versión para kindle.

Kessler, Suzanne (1998), Lessons from the intersexed. New Jersey, Rutgers University Press.

Lamas, Marta (2012) “Transexualidad: identidad y cultura”. México, Universidad Nacional Autónoma de México, tesis de doctorado en antropología.

Lamas, Marta (2014) “Transexualidad: ¿El estudio de lo extraño?”, en Cuerpo, sexo y política. México, Océano, pp. 135-155.

Machado, Paula, Angelo Brandelli, Henrique Caetano, Anna Vaitses, Igor Rabuske y Daniela Riva (2015), "Follow-up of psychological outcomes of interventions in patients diagnosed with disorders of sexual development: A systematic review”, en Journal of Health Psychology, SAGE, pp. 1-12. doi: $10.1177 / 1359105315572454$.

Mead, Margaret (1994 [1949]), Masculino y femenino. Madrid, Minerva Ediciones.

Money, John y Anke Ehrnardt (1972), Man and Woman: Boy and Girl: Differentiation and Dimorphism of Gender Identity from Conception to Maturity. Baltimore, Johns Hopkins University Press.

Nelson, Waldo, James McKay y Victor Vaughan (dir.) (1980), Tratado de pediatría, 7 a edición. México, Salvat.

OMS (Organización Mundial de la Salud) (1999), Trastornos mentales y del comportamiento de la decimal revisión de la Clasificación Internacional de Enfermedades CIE-10, consultado el 14 de septiembre de 2014, URL: http://es.slideshare.net/danielafigue/trastornosmentalescomportamiento-cie10.

OMS (Organización Mundial de la Salud) (2014), Eliminating forced, coercive and otherwise involuntary sterilization: An interagency statement, consultado el 10 de agosto de 2014, URL: http://www.who.int/reproductivehealth/publications/gender_rights/eliminating-forced-sterilization/en/.

Poisson, Jayme (2011), "Parents keep child's gender secret”, consultado el 15 de julio de 2015, URL: http://www.thestar.com/life/parent/2011/05/21/parents_keep_childs_gender_secret.html. 
Regueiro, Iñaki (2012), “El derecho a la identidad de género de niñas, niños y adolescentes”, en Revista Derechos Humanos, año 1, núm. 1, pp.101-115.

Stoller, Robert (1968), Sex and Gender: On the Development of Masculinity and Feminity. Nueva York, Science House.

Video A (2014), Vivo en Arg: Sociedad: La historia de Luana, “Yo nena, yo princesa”. Argentina, consultado el 28 de Julio de 2014, URL: https://www.youtube.com/watch?v=uLX2bKXV1ao.

Video B (2014), Presentación del libro: “Yo nena, yo princesa”. Argentina, consultado el 20 de agosto de 2015, URL: https://www.youtube.com/watch?v=zOuK81Rnw-0.

Wilson, Ian Chris Griffin y Bernadette Wren (2002), "The Validity of the Diagnosis of Gender Identity Disorder (Child and Adolescent Criteria)", en Clinical Child Psychology and Psychiatry, vol. 7, núm. 3, pp. 335-351.

Witterick, Kathy (2013), "Dancing in the Eye of the Storm", en Fiona Joy Green y May Friedman (eds.), Chasing Rainbows: Exploring Gender Fluid Parenting Practices. Canada, Demeter Press, versión para Kindle.

Wren, Bernadette (2014), "Thinking postmodern and practising in the enlightenment: Managing uncertainty in the treatment of children and adolescents”, en Feminism \& Psychology, vol. 24, núm. 2, pp. 271-291.

Fecha de recepción: 6 de septiembre de 2015

Fecha de aceptación: 27 de noviembre de 2015 